\title{
Respiratory Infections
}

\author{
Olga Klinkova
}

\begin{abstract}
Pneumonia is defined as the presence of a new pulmonary infiltrate on radiologic imaging in the patient with appropriate clinical symptoms such as fever, cough, production of purulent sputum, shortness of breath and/or hypoxia, in the absence of pulmonary edema $[1,2]$.

Keywords Pneumonia in neutropenic cancer patients · Community acquired viruses · Invasive fungal infection · Diffuse alveolar hemorrhage (DAH) · Cryptogenic organizing pneumonia (COP) $\cdot$ Consolidation pneumonia $\cdot$ Nodular and/or cavitary infiltrates · "Halo" sign · Reverse "halo" sign · Scattered nodules · Viral pneumonia $\cdot$ Rhinosinusitis
\end{abstract}

\section{Pneumonia/Pulmonary Infiltrates}

\section{Introduction}

Pneumonia is defined as the presence of a new pulmonary infiltrate on radiologic imaging in the patient with appropriate clinical symptoms such as fever, cough, production of purulent sputum, shortness of breath and/or hypoxia, in the absence of pulmonary edema $[1,2]$.

Absolute as well as functional neutropenia is the most important risk factor for development of pneumonia in cancer patients [3]. Duration of neutropenia is another important factor to consider as the etiology of pneumonia may differ in patients with prolonged ( $>15$ days) neutropenia. Additionally, in patients undergoing hematopoietic stem cell transplant (HCT), time lapsed after transplant, antimicrobial prophylaxis

O. Klinkova, MD $(\bowtie)$

Moffitt Cancer Center, Tampa, FL, USA

e-mail: olga.klinkova@moffitt.org 
and intensity of immunosuppression should be considered, as these variables predict the development of certain pneumonia types in this unique patient population.

\section{Microbiology}

\section{Bacterial Pathogens}

Similar to the general patient population, pneumonia in neutropenic cancer patients can be classified based on the mode of acquisition into community-acquired pneumonia (CAP), hospital-acquired pneumonia (HAP), and ventilator-associated pneumonia (VAP).

CAP is defined as the type of pneumonia that develops in patients who have not been recently hospitalized. A true CAP is infrequently observed in neutropenic patients. CAP may be considered in patients with chronic prolonged neutropenia residing in the community as well as in neutropenic patients with newly diagnosed hematologic malignancies starting active chemotherapy. The most frequent pathogen causing CAP in cancer patients is Streptococcus pneumoniae [3, 4]. Other bacterial pathogens in neutropenic cancer patients causing CAP include Haemophilus influenzae, Staphylococcus aureus and Pseudomonas aeruginosa. Atypical pathogens such as Legionella spp., Chlamydophila pneumoniae and Mycoplasma pneumoniae should be included into the differential diagnosis as well.

HAP is defined as a pneumonia that is not present at the time of admission and instead occurs 48 hours or more after admission, whereas VAP is defined as pneumonia occurring 48 hours or more after intubation [1].

HAP and VAP are caused by a broad spectrum of pathogens with gram-negative bacilli being the most prevalent in the neutropenic cancer patient population [5-7]. The organisms that should be always considered include Pseudomonas aeruginosa, Escherichia coli, Klebsiella pneumoniae, Enterobacter spp., Serratia spp. and Acinetobacter spp. [5, 7]. Staphylococcus aureus is the most common gram-positive pathogen causing HAP/VAP. Streptococcus pneumoniae, Haemophilus influenzae could also be encountered, especially in patients with minimal or no recent exposure to antibiotics or the hospital environment [3]. Isolation of certain gram-positive organisms from the blood or sputum samples, such as Viridans group streptococcus, Enterococcus spp., Bacillus spp., should be interpreted with caution as they are rarely implicated in the development of pneumonia. In such cases alternative source/ pathogens need to be considered [7]. Table 1 illustrates the list of the most commonly encountered bacterial pathogens in neutropenic patients.

The majority of neutropenic cancer patients are at risk for the development of multidrug resistant pathogens due to antimicrobial prophylaxis use, extensive exposure to broad-spectrum antibiotics as well as the nosocomial environment [8, 9]. Forty to eighty percent of Staphylococcus aureus isolates are methicillin-resistant [9]. The most important mechanisms of gram-negative organism resistance include the production of various beta-lactamases such as Ambler class C (AmpC) beta-lactamases and extended-spectrum beta-lactamases (ESBL). The emergence of carbapenem- resistant 
Table 1 Etiologic agents of pneumonia in neutropenic patients

\begin{tabular}{l|l|l}
\hline Bacterial & Viral & Fungal \\
\hline Gram-positive & Influenza A/B virus & Aspergillus spp. \\
Streptococcus pneumoniae & Parainfluenza virus & Zygomycetes \\
Staphylococcus aureus & Human metapneumovirus virus & Fusarium spp. \\
Beta-hemolytic Streptococcus & RSV & Scedosporium spp. \\
Streptococcus Viridans & Adenovirus & Dark-walled fungi \\
Rhodococcus equi & CMV & Histoplasma capsulatum \\
Gram-negative & Coccidioides immitis \\
Haemophilus influenzae & HSV & Blastomyces dermatididis \\
Moraxella Catarrhalis & HHV-6 & Pneumocystis jiroveci \\
Pseudomonas Aeruginosa & & \\
Escherichia Coli & & \\
Klebsiella Pneumoniae & & \\
Enterobacter cloacae & & \\
Serratia spp. & \\
Proteus spp. & \\
Acinetobacter baumanii complex & & \\
Stenotrophomonas maltophila & & \\
Burkholderia spp. & & \\
Atypical bacteria & \\
Legionella spp. & \\
Chlamydophila pneumoniae & & \\
Mycoplasma pleumoniae & & \\
$\begin{array}{l}\text { Mycobacteria } \\
\text { Mycobacterium tuberculosis }\end{array}$ & & \\
Nontuberculous mycobacerium & & \\
Other pathogens & & \\
Nocardia spp. & & \\
Actynomyces spp. & & \\
\hline
\end{tabular}

Enterobacteriaceae (CRE) is concerning. It has been associated with a delay in necessary appropriate antibiotic therapy and high mortality in cancer patients [10].

\section{Viral Pathogens}

Community-acquired viruses such as enterovirus/rhinovirus, influenza, respiratory syncytial virus (RSV) and other community-acquired viruses are commonly seen in neutropenic patients [3, 7]. In patients post HSCT and other severely immunocompromised patients, cytomegalovirus (CMV), varicella zoster virus (VZV) and, less commonly, HHV-6 should be considered as etiologic agents along with other viruses $[7,11]$ (Table 1).

\section{Fungal Pathogens}

In patients with profound $(\mathrm{ANC}<500)$ and/or prolonged duration of neutropenia ( $>10$ days), in addition to the above mentioned etiologies, fungal pathogens should be strongly considered [12]. Aspergillus fumigatus is the most common fungal pathogen 
causing pneumonia in this patient population. Aspergillus spp., Zygomycetes, Fusarium spp., Pseudaalesheria boydii, and dark-walled fungi are other potential etiologies that cause fungal pneumonia [3,12, 13]. Endemic fungal pneumonias caused by Histoplasma capsulatum, Coccidioides immitis and Blastomyces dermatididis can occasionally occur, but are less common in neutropenic patients [3, 12].

Neutropenic patients with associated CD4 + cell depletion not receiving appropriate prophylaxis are at high risk for Pneumocystis jiroveci pneumonia [14, 15]. Examples include patients with acute lymphoblastic leukemia, those receiving CD4 + cell-depleting chemotherapy or chronic corticosteroids (equivalent of $20 \mathrm{mg} /$ day prednisone for more than 1 month).

\section{Other Pathogens}

Rhodococcus, Actynomyces spp., Nocardia spp. and Mycobacterium spp. should be also considered $[7,16]$ in the etiology of pneumonia in neutropenic patients, especially with a subacute presentation.

\section{Clinical Manifestations}

Fever, increased production of purulent sputum, dyspnea, and decrease in oxygenation along with the findings of a new infiltrate are signs of pneumonia. In neutropenic patients, the symptoms can be rather subtle or atypical. Pleuritic chest pain and hemoptysis may be present as well.

\section{Differential Diagnosis}

The differential diagnosis of pulmonary infiltrates in a neutropenic patient is broad. Other etiologies that should be considered include acute respiratory distress syndrome (ARDS), transfusion related lung injury (TRALI), diffuse alveolar hemorrhage(DAH), drug and radiation toxicity or pneumonitis, cryptogenic organizing pneumonia (COP), cardiogenic and non-cardiogenic pulmonary edema from capillary leak and the underlying malignancy $[2,17]$.

\section{Diagnosis and Radiologic Findings}

Chest radiograph has a low sensitivity for early detection of pulmonary infiltrates in febrile neutropenic patients [17-19].This is especially true given the broad differential diagnosis discussed above Therefore, chest computed tomography (CT) is now considered the standard imaging procedure for diagnosing pneumonia in this patient 
Table 2 Key radiologic features of pneumonia caused by different fungal agents

\begin{tabular}{l|l}
\hline Fungal organism & Radiologic appearance \\
\hline Aspergillus spp. & $\begin{array}{l}\text { Few or single segmental nodular consolidations (diameter }>1-2 \mathrm{~cm}) \\
\text { Few or single ground glass opacities with lobular distributions } \\
\text { Infiltrates mostly peripherally located } \\
\text { Nodular infiltrate with surrounded by ground glass infiltrate "Halo" sign }\end{array}$ \\
\hline Zygomycetes & $\begin{array}{l}\text { Nodular consolidations (diameter }>2 \mathrm{~cm}), \text { mostly peripherally located } \\
\text { Ground glass surrounded by an area of consolidation Reverse "Halo" sign. } \\
\text { With or without } \\
\text { Pleural effusion }\end{array}$ \\
\hline $\begin{array}{l}\text { Fusarium spp. } \\
\text { Scedosporium } \\
\text { spp. }\end{array}$ & Scattered nodular consolidations (multiple $(>2)$, average diameter $<1 \mathrm{~cm})$ \\
\hline
\end{tabular}

population. The number, characteristics and distribution of the infiltrates can help with the differential diagnosis and guide with a choice of empiric antimicrobial therapy (Table 2).

Consolidation pneumonia can be caused by bacterial pathogens such as s. aureus, pseudomonas, and enterobacteriaceae. Legionella can cause ground glass or consolidation pneumonia (Fig. 1). Diffuse bilateral infiltrates and bilateral ground-glass attenuation should raise clinical suspicion for atypical pathogens, viral (Fig. 2), or PJ pneumonia (Fig. 3) or a non-infectious etiology [18] (Fig. 4).

Nodular and/or cavitary infiltrates indicate the possibility of invasive fungal infection (IFI), especially in patients with prolonged duration of neutropenia ( $>10$ days). The "halo" sign is defined as a nodule (typically $>1 \mathrm{~cm}$ ) surrounded by a perimeter of ground-glass opacity representing hemorrhage [20] (Fig. 5) This sign is commonly seen in patients with invasive pulmonary aspergillosis (IPA) [17, 21]. The reverse "halo" sign, defined as a focal area of ground glass attenuation surrounded by consolidation (Fig. 6) is more frequently observed with zygomycosis $[22,23]$. Fusarium pulmonary infections are typically characterized by the presence of multiple small $(<1 \mathrm{~cm})$ scattered nodules [23].

To establish a microbiologic diagnosis, sputum and blood cultures should be obtained. Streptococcus pneumoniae, Legionella urinary antigens and the respiratory viral panel PCR panel can assist with rapid diagnosis if positive. When IPA is suspected, it is strongly recommended to obtain serum galactomannan (GM) in patients with hematologic malignancies [18, 20].

Unless the etiology of pneumonia was established in an expedited manner using non-invasive rapid diagnostic testing or culture method as indicated above, bronchoscopy with bronchoalveolar lavage (BAL) for cultures should be strongly considered in a febrile neutropenic patient with new pulmonary infiltrates [18, 24, 25]. Active bleeding or severe hypoxia might not allow early bronchoscopy, but it is generally considered a safe procedure in this patient population [24]. BAL sample should be sent for "standard", fungal, mycobacterial, nocardia, Legionella cultures, respiratory viral panel and cytology. Based on prior studies, measurement of BAL GM may offer even higher sensitivity comparing to the performance of blood GM assays for the diagnosis of invasive fungal pneumonia $[12,13]$. 
Fig. 1 Chest CT of a patient with Legionella pneumonia

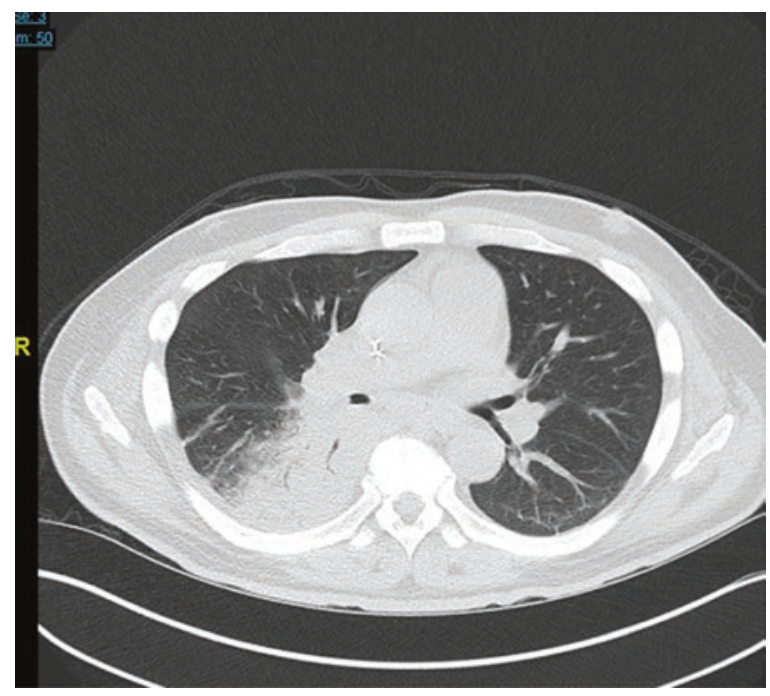

Fig. 2 Chest CT of a patient with RSV pneumonia

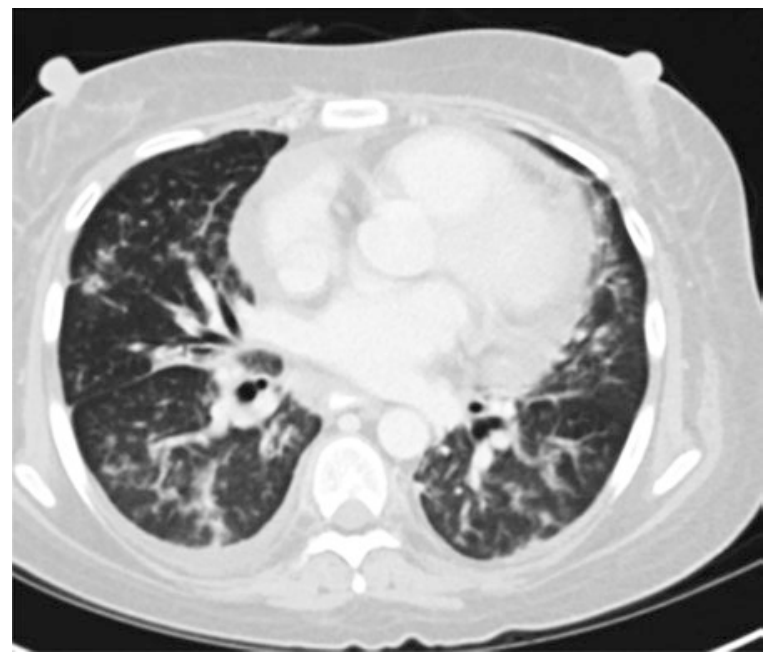

Trans bronchial biopsy is rarely used in this patient population due to severe thrombocytopenia and risk of complications such as a life-threatening bleeding. CT-guided percutaneous lung biopsy can be considered for the patient with large peripheral lesions if the diagnosis is expected to change medical management and the benefits of the procedure outweigh its risks. 


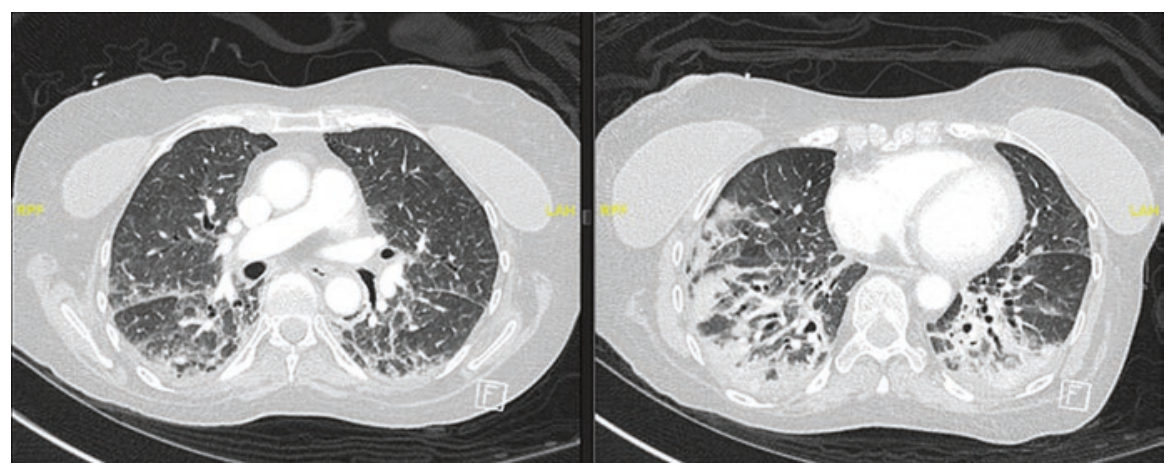

Fig. 3 Chest CT of a patient with PJP pneumonia

Fig. 4 Chest CT of a patient with DAH

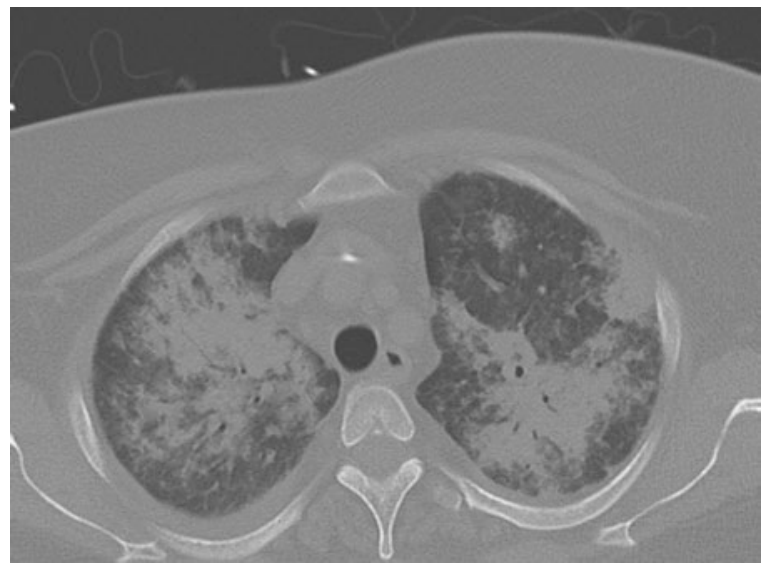

\section{Treatment}

Antibiotic therapy should be initiated as soon as the blood and sputum cultures are obtained, but should not be held if there is a delay in obtaining a diagnostic work-up.

Initial empiric antibiotic regimens of a newly diagnosed pneumonia in a febrile neutropenic patient should include broad-spectrum antibiotic agents to cover Pseudomonas and S. aureus (including MRSA) [26]. Patients at risk for multidrugresistant pathogens or with a prior history of such, as well as patients at high risk for mortality at the time of presentation (septic shock or pneumonia requiring ventilator support) should be prescribed two anti-pseudomonal agents from different classes until further information from the cultures is available [1]. Beta-lactam antibiotics with anti-pseudomonal coverage include cefepime, ceftazidime, piperacillintazobactam, or a carbapenem with anti-pseudomonal activity such as meropenem or imipenem. Aztreonam can be used as an alternative agent in patients with a documented penicillin allergy. A second anti-pseudomonal agent can be either an 
Fig. 5 CT chest with the halo sign

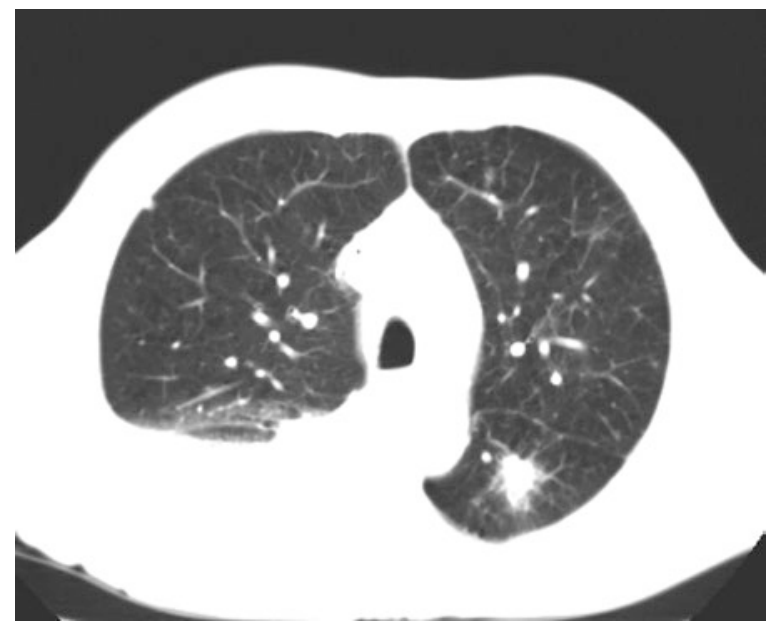

Fig. 6 Chest $\mathrm{CT}$ with the reverse halo sign

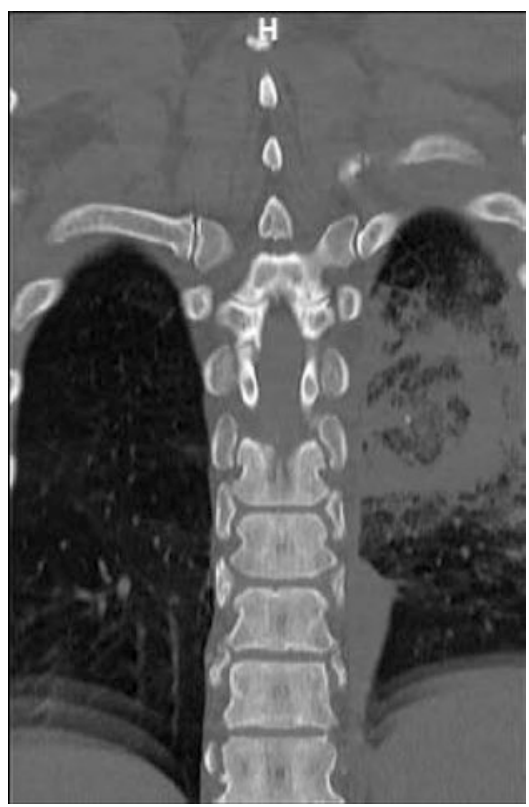

aminoglycoside or a fluoroquinolone; however, neither of these antibiotics should be used as sole agents. Aminoglycoside monotherapy for the treatment of pneumonia is not recommended due to poor lung penetration and alveolar concentration, prompting higher antibiotic dosing that potentially can lead to nephro- and ototoxicity $[1,27]$. Fluoroquinolone monotherapy is discouraged due to the increasing rate of resistance to this particular antibiotic class. Agents with MRSA coverage, vancomycin or linezolid, should also be included into the initial treatment regimen of pneumonia. 
In neutropenic cancer patients with a true community-acquired pneumonia or an early hospital acquired pneumonia, an agent with coverage against atypical pathogens such as Legionella spp., Mycoplasma spp. and Chlamydophila pneumoniae should be included into the regimen. Fluoroquinolones, doxycycline or azithromycin should be a part of the regimen in such clinical scenarios [28].

Anti-fungal therapy should be initiated in neutropenic patients with high suspicion for invasive fungal pneumonia on radiographic testing or in the patients that do not appropriately respond to the initial antibiotic regimen, [3, 18]. Voriconazole or liposomal amphotericin B remains the preferred first-line regimen. In patients with suspected mucormycosis, liposomal amphotericin B is the preferred first-line agent for the initial therapy. Primary monotherapy with echinocandins is not recommended due to low treatment response rates in patients with IPA [13, 20]. Combination regimens with two different anti-fungal agents are typically reserved as a salvage therapy in the treatment of IPA and mucormycosis $[3,13,20]$.

In patients with suspected PJ pneumonia, first line therapy with high-dose trimethoprim-sulfamethoxazole (TMP/SMX) should be initiated [18, 29]. In patients with allergic reactions or contraindications to TMP/SMX, a combination therapy with clindamycin plus primaquine may be considered as an alternative [18, 29]. Routine adjunctive therapy with steroids in non-HIV patients with the diagnosis of PJ pneumonia is not recommended [18, 29, 30].

Treatment of viral pneumonia is based on the etiologic agent. CMV pneumonia is treated with intravenous gancyclovir or foscarnet with a combination of intravenous immunoglobulin or CMV-specific immunoglobulin [18, 31, 32]. Due to the side effect of pancytopenia, ganciclovir is generally contraindicated in the settings of severe neutropenia or a pre-engraftment phase of HSCT [31, 32]. For the treatment of influenza A and B infections, neuraminidase inhibitors, such as oseltamivir, zanamivir, or peramivir are recommended [31]. RSV pneumonia is typically treated with aerosolized, oral or intravenous ribavirin [31, 33, 34]. Addition of intravenous immunoglobulin may further reduce mortality in this patient population [35]. In high-risk patients with severe human metapneumovirus (hMPV) pneumonia, ribavirin treatment may be used as well but the use of this agent remains controversial [31, 36]. For the treatment of parainfluenza virus (PIV), the use of ribavirin has been described in the literature; albeit without any evidence of reduced mortality [37]. Treatment with interferon Alpha-2b for severe PIV pneumonia may be another promising therapeutic option that needs to be studied further [38].

\section{Rhinosinusitis}

\section{Introduction}

Rhinosinusitis is defined as inflammation of the nasal cavity and paranasal sinuses [39]. Neutropenic patients with cancer are at increased risk for unusual microorganisms as a cause of rhinosinusitis as well as development of invasive or complicated disease. 


\section{Microbiology}

In a healthy (not immune-compromised) host, community-acquired viruses such as rhinovirus, influenza and parainfluenza are responsible for the development of sinusitis [40] and should be considered in neutropenic patients with this condition as well. When a bacterial infection occurs, causative organisms to consider in this patient population are Pseudomonas aeruginosa, Staphylococcus aureus, Streptococcus pneumoniae, Haemophilus influenzae, and Moraxella catarrhalis $[41,42]$. Oral anaerobic flora such as Prevotella spp., Fusobacterium spp. and Peptostreptococcus spp. are implicated as well, especially in chronic sinusitis [40]. Fungal/mold pathogen such as Aspergillus spp., mucorales, Fusarium spp., and dematiaceous fungi are the causes of invasive fungal sinusitis, commonly seen in patients with prolonged neutropenia [43].

\section{Clinical Manifestations}

Purulent nasal drainage, nasal congestion, facial pain and pressure are the most common signs of acute sinusitis [39, 41]. Fever might be present in some of the patients. In complicated sinusitis, the infection might spread beyond the paranasal sinuses and nasal cavity. In such cases, oculo-orbital and CNS symptoms such as periorbital edema, erythema, vision changes, cranial nerve palsy, meningismus or focal neurologic symptoms might occur. The presence of necrotic eschars in the oral or nasal cavities should raise a high suspicion for invasive fungal process.

\section{Diagnosis}

In febrile neutropenic patient with acute sinusitis, CT sinuses is typically the first diagnostic modality and can evaluate the extent of sinus involvement including possible fungal bony erosion or orbital invasion [44]. (Fig. 7).MRI sinuses could be used as an alternative imaging technique or if intracranial or cavernous sinus involvement is suspected. Early direct endoscopic visualization of the nasal mucosa by an otolaryngologist should be pursued [45]. Endoscopic biopsies and cultures of the affected tissues are typically positive to establish the diagnosis.

Nasopharyngeal swab for a respiratory viral panel aids in the diagnosis of uncomplicated viral sinusitis. Nasal swab cultures are not recommended due to their poor reliability. 
Fig. 7 CT sinuses of a patient with mold sinusitis

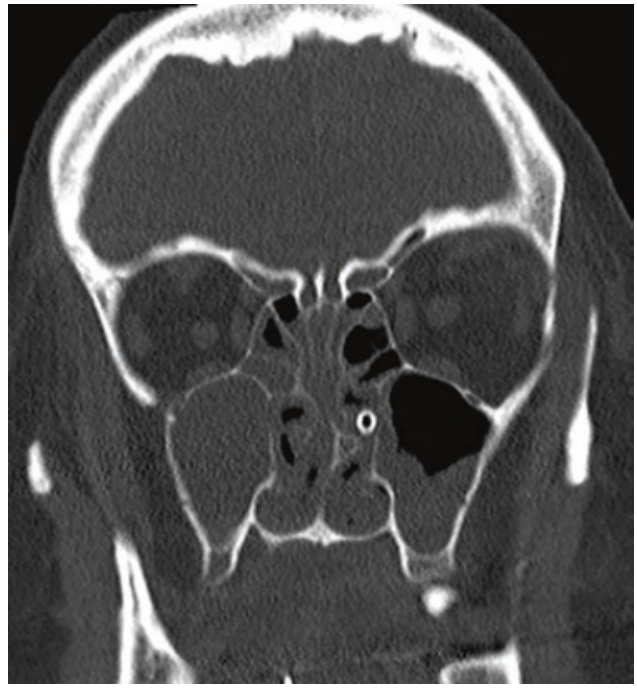

\section{Treatment}

Febrile neutropenic patients with diagnosis of acute bacterial sinusitis should receive broad-spectrum antibiotics with anti-pseudomonal activity until culture results become available. These antibiotic options include cefepime, ceftazidime, piperacillin-tazobactam or an anti-pseudomonal carbapenem such as meropenem, imipenem or doripenem. The decision to include anti-MRSA agent, such as vancomycin or linezolid, should be based on patient-specific risk factors and the severity of the illness.

When invasive fungal sinusitis is suspected in a patient with prolonged neutropenia, treatment should not be delayed. Voriconazole, isavuconazole or liposomal amphotericin B are first-line options. In patients with high suspicion for invasive mucormycosis, liposomal amphotericin B is considered to be the drug of choice for initial therapy. Early surgical debridement by an otolaryngologist must be considered in such cases.

\section{References}

1. Kalil AC, et al. Management of adults with hospital-acquired and ventilator-associated pneumonia: 2016 clinical practice guidelines by the Infectious Diseases Society of America and the American Thoracic Society. Clin Infect Dis. 2016;63(5):e61-e111.

2. Vehreschild JJ. Pneumonia and lung infiltrates in neutropenic patients: many stones unturned. Ann Am Thorac Soc. 2013;10(5):493-5.

3. Evans SE, Ost DE. Pneumonia in the neutropenic cancer patient. Curr Opin Pulm Med. 2015;21(3):260-71. 
4. Sousa D, et al. Community-acquired pneumonia in immunocompromised older patients: incidence, causative organisms and outcome. Clin Microbiol Infect. 2013;19(2):187-92.

5. American Thoracic Society, Infectious Diseases Society of America. Guidelines for the management of adults with hospital-acquired, ventilator-associated, and healthcare-associated pneumonia. Am J Respir Crit Care Med. 2005;171(4):388-416.

6. Lossos IS, et al. Bacterial pneumonia in recipients of bone marrow transplantation. A five-year prospective study. Transplantation. 1995;60(7):672-8.

7. Rolston KV. The spectrum of pulmonary infections in cancer patients. Curr Opin Oncol. 2001;13(4):218-23.

8. Perez F, Adachi J, Bonomo RA. Antibiotic-resistant gram-negative bacterial infections in patients with cancer. Clin Infect Dis. 2014;59(Suppl 5):S335-9.

9. Rapoport B, et al. The emerging problem of bacterial resistance in cancer patients; proceedings of a workshop held by MASCC "Neutropenia, Infection and Myelosuppression" Study Group during the MASCC annual meeting held in Berlin on 27-29 June 2013. Support Care Cancer. 2016;24(7):2819-26.

10. Satlin MJ, et al. Emergence of carbapenem-resistant Enterobacteriaceae as causes of bloodstream infections in patients with hematologic malignancies. Leuk Lymphoma. 2013;54(4):799-806.

11. Konoplev S, et al. Cytomegalovirus pneumonia in adult autologous blood and marrow transplant recipients. Bone Marrow Transplant. 2001;27(8):877-81.

12. Young AY, Leiva Juarez MM, Evans SE. Fungal pneumonia in patients with hematologic malignancy and hematopoietic stem cell transplantation. Clin Chest Med. 2017;38(3):479-91.

13. Mulanovich VE, Kontoyiannis DP. Fungal pneumonia in patients with hematologic malignancies: current approach and management. Curr Opin Infect Dis. 2011;24(4):323-32.

14. Souza JP, et al. High rates of pneumocystis carinii pneumonia in allogeneic blood and marrow transplant recipients receiving dapsone prophylaxis. Clin Infect Dis. 1999;29(6):1467-71.

15. Yale SH, Limper AH. Pneumocystis carinii pneumonia in patients without acquired immunodeficiency syndrome: associated illness and prior corticosteroid therapy. Mayo Clin Proc. 1996;71(1):5-13.

16. Henkle E, Winthrop KL. Nontuberculous mycobacteria infections in immunosuppressed hosts. Clin Chest Med. 2015;36(1):91-9.

17. Maschmeyer G. Pneumonia in febrile neutropenic patients: radiologic diagnosis. Curr Opin Oncol. 2001;13(4):229-35.

18. Maschmeyer G, et al. Diagnosis and antimicrobial therapy of lung infiltrates in febrile neutropenic patients (allogeneic SCT excluded): updated guidelines of the infectious diseases working party (AGIHO) of the German Society of Hematology and Medical Oncology (DGHO). Ann Oncol. 2015;26(1):21-33.

19. Maschmeyer G, et al. Diagnosis and antimicrobial therapy of lung infiltrates in febrile neutropenic patients: guidelines of the infectious diseases working party of the German Society of Haematology and Oncology. Eur J Cancer. 2009;45(14):2462-72.

20. Patterson TF, et al. Practice guidelines for the diagnosis and management of aspergillosis: 2016 update by the Infectious Diseases Society of America. Clin Infect Dis. 2016;63(4):e1-e60.

21. Satish Kumar Bajaj BT. Respiratory infections in immunocompromised patients: lung findings using chest computed tomography. Radiol Infect Dis. 2017;4:29-37.

22. Wahba $\mathrm{H}$, et al. Reversed halo sign in invasive pulmonary fungal infections. Clin Infect Dis. 2008;46(11):1733-7.

23. Farooq A, Alrabaa S, Quilitz R, Yacoub A, Maroon ME, Fulp W, Sandin R, Greene JN. Comparison of clinical and radiological features of aspergillus, zygomycosis, and fusarium pneumonia in neutropenic patients. Infect Dis Clin Pract. 2014;22(5):288-93.

24. Hohenadel IA, et al. Role of bronchoalveolar lavage in immunocompromised patients with pneumonia treated with a broad spectrum antibiotic and antifungal regimen. Thorax. 2001;56(2):115-20. 
25. Kuehnhardt D, et al. Therapeutic implication of BAL in patients with neutropenia. Ann Hematol. 2009;88(12):1249-56.

26. Freifeld AG, et al. Clinical practice guideline for the use of antimicrobial agents in neutropenic patients with cancer: 2010 update by the infectious diseases society of america. Clin Infect Dis. 2011;52(4):e56-93.

27. Carcas AJ, et al. Tobramycin penetration into epithelial lining fluid of patients with pneumonia. Clin Pharmacol Ther. 1999;65(3):245-50.

28. Mandell LA, et al. Infectious Diseases Society of America/American Thoracic Society consensus guidelines on the management of community-acquired pneumonia in adults. Clin Infect Dis. 2007;44(Suppl 2):S27-72.

29. Maschmeyer G, et al. ECIL guidelines for treatment of pneumocystis jirovecii pneumonia in non-HIV-infected haematology patients. J Antimicrob Chemother. 2016;71(9):2405-13.

30. Lemiale V, et al. Adjunctive steroid in HIV-negative patients with severe pneumocystis pneumonia. Respir Res. 2013;14:87.

31. Vakil E, Evans SE. Viral pneumonia in patients with hematologic malignancy or hematopoietic stem cell transplantation. Clin Chest Med. 2017;38(1):97-111.

32. Marchesi $\mathrm{F}$, et al. Cytomegalovirus infection in hematologic malignancy settings other than the allogeneic transplant. Hematol Oncol. 2018;36(2):381-91.

33. Khanna N, et al. Respiratory syncytial virus infection in patients with hematological diseases: single-center study and review of the literature. Clin Infect Dis. 2008;46(3):402-12.

34. Weigt SS, et al. Respiratory viral infections in hematopoietic stem cell and solid organ transplant recipients. Semin Respir Crit Care Med. 2011;32(4):471-93.

35. Torres HA, et al. Characteristics and outcome of respiratory syncytial virus infection in patients with leukemia. Haematologica. 2007;92(9):1216-23.

36. Waghmare A, Englund JA, Boeckh M. How I treat respiratory viral infections in the setting of intensive chemotherapy or hematopoietic cell transplantation. Blood. 2016;127(22):2682-92.

37. Chemaly RF, et al. The characteristics and outcomes of parainfluenza virus infections in 200 patients with leukemia or recipients of hematopoietic stem cell transplantation. Blood. 2012;119(12):2738-45; quiz 2969.

38. Marinez J, Vincent AL, Sandin RL, Greene JN. Interferon Alfa-2b as a successful treatment for parainfluenza virus pneumonia in a non-hodgkin lymphoma patient. Infect Dis Clin Pract. 2008;16(3):187-9.

39. Rosenfeld RM. CLINICAL PRACTICE. Acute sinusitis in adults. $\mathrm{N}$ Engl J Med. 2016;375(10):962-70.

40. Brook I. Microbiology of sinusitis. Proc Am Thorac Soc. 2011;8(1):90-100.

41. Rosenfeld RM, et al. Clinical practice guideline (update): adult sinusitis. Otolaryngol Head Neck Surg. 2015;152(2 Suppl):S1-S39.

42. Ito CJ, Jackson RS, Castro-Borobio M, Nanjappa S, Klinkova O, Phommachanh V, Keeler JA, Greene JN, Padhya TA, Tabor MH. Microbiology of acute rhinosinusitis in immunosuppressed patients. Infect Dis Clin Pract. 2017;25:260-3.

43. Davoudi $\mathrm{S}$, et al. Invasive mould sinusitis in patients with haematological malignancies: a 10 year single-centre study. J Antimicrob Chemother. 2015;70(10):2899-905.

44. DelGaudio JM, et al. Computed tomographic findings in patients with invasive fungal sinusitis. Arch Otolaryngol Head Neck Surg. 2003;129(2):236-40.

45. DelGaudio JM, Clemson LA. An early detection protocol for invasive fungal sinusitis in neutropenic patients successfully reduces extent of disease at presentation and long term morbidity. Laryngoscope. 2009;119(1):180-3. 